\title{
The Intervention Threshold for Intracranial Pressure of Traumatic Brain Injury Patients Can Be Determined by Clustering Algorithms and Is Observed to Be $13 \mathrm{~mm} \mathrm{Hg}$
}

\author{
Hermann Prossinger, ${ }^{1, *}$, Hubert Hetz ${ }^{2}$, Alexandra Acimovic ${ }^{2}$, Reinhard Berger ${ }^{2}$, Karim Mostafa $^{3}$, \\ Alexander Grieb ${ }^{3}$, Heinz Steltzer ${ }^{2,3}$ \\ ${ }^{1}$ Department of Evolutionary Anthropology, Faculty of Life Sciences, University of Vienna, Vienna, Austria \\ ${ }^{2}$ Department for Anaesthesiology and Intensive Medical Care, Trauma Center Vienna, Location Meidling, Vienna, Austria \\ ${ }^{3}$ Faculty of Medicine, Sigmund Freud Private University, Vienna, Austria
}

Email address:

hermann.prossinger@univie.ac.at (H. Prossinger)

${ }^{*}$ Corresponding author

\section{To cite this article:}

Hermann Prossinger, Hubert Hetz, Alexandra Acimovic, Reinhard Berger, Karim Mostafa, Alexander Grieb, Heinz Steltzer. The Intervention Threshold for Intracranial Pressure of Traumatic Brain Injury Patients Can Be Determined by Clustering Algorithms and Is Observed to Be 13 mm Hg. Clinical Medicine Research. Vol. 8, No. 1, 2019, pp. 6-15. doi: 10.11648/j.cmr.20190801.12

Received: January 21, 2019; Accepted: February 22, 2019; Published: March 12, 2019

\begin{abstract}
During treatment in an intensive care unit (ICU), traumatic brain injury (TBI) patients sometimes suffer an increase in intracranial pressure (ICP). An increase beyond a currently unknown and to-be-determined threshold is very often life-threatening and requires intervention by the clinical staff. Because this threshold value is considered unknown, 'conventional wisdom' of practitioners argue it to be $20 \mathrm{~mm} \mathrm{Hg}$. No published studies include statistical methods that could supply a rigorous outcome for the threshold value. Here, we use a clustering algorithm ( $K$-means clustering) to find threedimensional clusters of the 984 triples of ICP, temperature and patient state index (PSI, a proxy for sedation level). The algorithm outputs three clusters and two gaps. One gap separates two clusters from a third and is almost planar, and perpendicular to the ICP axis (implying a threshold across all temperatures and all sedation levels); the other is perpendicular to the temperature axis, which terminates at the aforementioned gap. The first gap provides a statistically rigorous threshold of $13.625 \mathrm{~mm} \mathrm{Hg}$ for ICP intervention. The second gap defines a threshold temperature $\left(36.5^{\circ} \mathrm{C}\right)$. The gap between the two temperature regimes does not continue into Cluster 3, implying that the intervention threshold for ICP is independent of temperature.
\end{abstract}

Keywords: Intracranial Pressure, Traumatic Brain Injury, Clustering Algorithms, Patient State Index, Akaike's Information Criterion, ICP Intervention Threshold, K-means Clustering

\section{Introduction}

Patients being treated for traumatic brain injury (TBI) occasionally suffer an elevated intracranial pressure (ICP), requiring intervention, because an elevated ICP is a medical emergency. [1] The criterion of when ICP is 'elevated' obviously needs to be known; otherwise, a necessary medical intervention may be missed. Current literature defines the threshold for elevated ICP as $20 \mathrm{~mm} \mathrm{Hg}$ [2-6] or higher (up to $30 \mathrm{~mm} \mathrm{Hg}$ [7]). We note that the most recent review of the ICP threshold guidelines relies on "conventional wisdom" to justify this threshold, [8, 9] rather than on statistical methodology, as Level I evidence is lacking. [10] The $20 \mathrm{~mm}$ $\mathrm{Hg}$ value is claimed to be related to observed increased mortality. [11] However, as many vital parameters are interrelated with ICP, we infer that looking at increased mortality via univariate statistics is fraught with unreliability. The interrelation between vital parameters necessitates a multivariate approach. Because some vital parameters are strongly correlated, we initially rely on ICU clinicians' observations that ICP, body temperature and sedation level are the ones that are least straightforwardly controllable in an ICU setting. When they vary, many correlated vital 
parameters vary as a result. As we will show in this paper, the clustering algorithm that we use indicates that body temperature and sedation level are quite independent of each other and of ICP only below a certain level, which we label $\mathrm{ICP}_{\text {threshold. }}$.

Because the volume of the intracranial cavity is fixed, any increase in ICP beyond some threshold will cause secondary brain injury. We investigate, in this paper, whether this threshold depends on the other two physiological parameters temperature and sedation level monitored by clinicians while the patient is being treated in an ICU. For reasons that are supported by the outcomes of the statistical analyses we describe below, we look at body temperature ${ }^{12}$ and patient state index (PSI [13, 14]) — a proxy for consciousness/unconsciousness (as well as a degree of sedation [15]).

In this paper, we introduce a statistical approach to determine a possibly new threshold for ICP. We find that 20 $\mathrm{mm} \mathrm{Hg}$ is far too high and argue that lowering the threshold value to slightly above $13 \mathrm{~mm} \mathrm{Hg}$ is necessary. We also investigate how this threshold depends (or not) on temperature. Our statistical approach shows a further finding: that the threshold for elevated ICP does not (in a statistical sense) depend on PSI.

\section{Materials}

\subsection{Patients}

The data set consists of 32 TBI patients treated in a neurotrauma intensive care unit (ICU) in Vienna (Trauma Center Vienna, Austria): 26 males and 6 females aged 21-80 years when they suffered a TBI (Table 1).

Table 1. Patient data. The age of each patient at time of event causing TBI has been calculated to the nearest day, as both the date of birth and the date of injury have been documented (we note that otherwise coincidental birth and accident dates may introduce an error of \pm 1 year). Sex is listed, not gender, because we do not know the gender identity of the patients, but we do know their biological sex. The ages are beta-distributed, hence we refrain from calculating standard deviations; rather, we list the modes, the expectation values, the $\pm 34 \cdot 1 \%$ range about the mode (see Figure 1 ), and the extremes. Using Wilks' 1 , we cannot exclude that the two distributions are drawn from the same population (details: see text). Expect is the expectation value of the distribution.

\begin{tabular}{|c|c|c|c|c|}
\hline Sex & Mode (years) & Expect (years) & $\pm 34.1 \%$ Interval about the mode (years) & Min-Max (years) \\
\hline Male & 45.1 & 46.1 & $28.8-61.2$ & $21.0-80.0$ \\
\hline Female & 61.5 & 60.5 & $51.6-71.6$ & $45.0-73.2$ \\
\hline
\end{tabular}

\subsection{Measured and Recorded Variables}

The date of birth and date of the event that led to TBI have been recorded for each patient.

Patients' intracranial pressure (ICP), body temperature (TEMP), and patient state index (PSI) were monitored for intervention purposes. ICP, TEMP and PSI readings were taken at 4 time points each day (6 am, noon, $6 \mathrm{pm}$ and midnight) and entered into the data set for subsequent analyses. Some triples $(4.7 \%)$ are incomplete or missing; due to therapeutic interventions taking place when the values are to be recorded, one or more of the 3 values had not been entered at the times noted above. In total, 984 triples were complete and used in the analyses. The data were stored in a patient data management system (PDMS COPRA ${ }^{\mathbb{B}}$ ).

\section{Ethics}

This study was approved by The Ethics Committee of the Trauma Center Vienna. As the SHT patients were unconscious during their treatment in the ICU, their agreement to the terms of this study could not be requested from them.

\section{Methods}

\subsection{Measurement Methods}

Intracranial Pressure (ICP) was measured with a Spiegelberg parenchymal catheter utilizing an air bladder technology (Spiegelberg GmbH \& Co. KG, Hamburg, Germany). Continuous body core temperature monitoring was performed using a Rüsch ${ }^{\mathrm{TM}}$ Sensor (Teleflex Medical, Ireland) with a catheter for urinary drainage. Patient sedation depth was obtained from a processed EEG parameter provided by SEDLine ${ }^{\circledR}$ brain function monitoring (MASIMO, Irvine, CA, USA); the (stored) outcome was the Patient State Index (PSI). [16]

\subsection{Statistical Analysis Methods}

\subsubsection{Distribution of Ages}

Ages are never normally distributed, because the domain of all possible ages is $[0, \max ] \in \mathfrak{R}$, with $\max \approx 1.2$ centuries worldwide. We calculate the Julian days between birth date and date of accident and convert to centuries; in our study we use $\max =1.0$ centuries (because no patient was older than 100 years). The domain of the distribution is thus $[0,1] \in \Re$, the most likely (ML) Beta distribution enables the determination of mode, expectation value, and the $\pm 34.1 \%$ uncertainty interval (Figure 1) for males and females separately or for the sample with the sexes pooled.

We use the log-likelihood ratio test to determine, via Wilks' $\Lambda$, whether the statistical populations from which the male and female samples are drawn are significantly different. [17-19] As we find that male and female samples are not significantly different, we continue our analysis with the pooled sample of (ICP, TEMP, PSI) triples, irrespective of age and (biological) sex of the TBI patients. In this analysis, we do not look at the temporal evolution of the ICP per patient. We use only those triples for which all 3 values had 
been registered: a total of 984 points in 3D (horizontal axes: ICP, and TEMP; vertical axis: PSI), regardless of patient or registration time.

All statistical analyses in this paper use non-symmetric distributions, so (a) the expectation value of the distribution differs from its mode, and (b) the conventional \pm SD interval is not meaningful. We therefore compute asymmetric boundaries about the mode with probabilities $\pm 34.1 \%$ (equal to the probability of $\pm 1 \mathrm{SD}$ from the mode for a normal/Gaussian distribution).

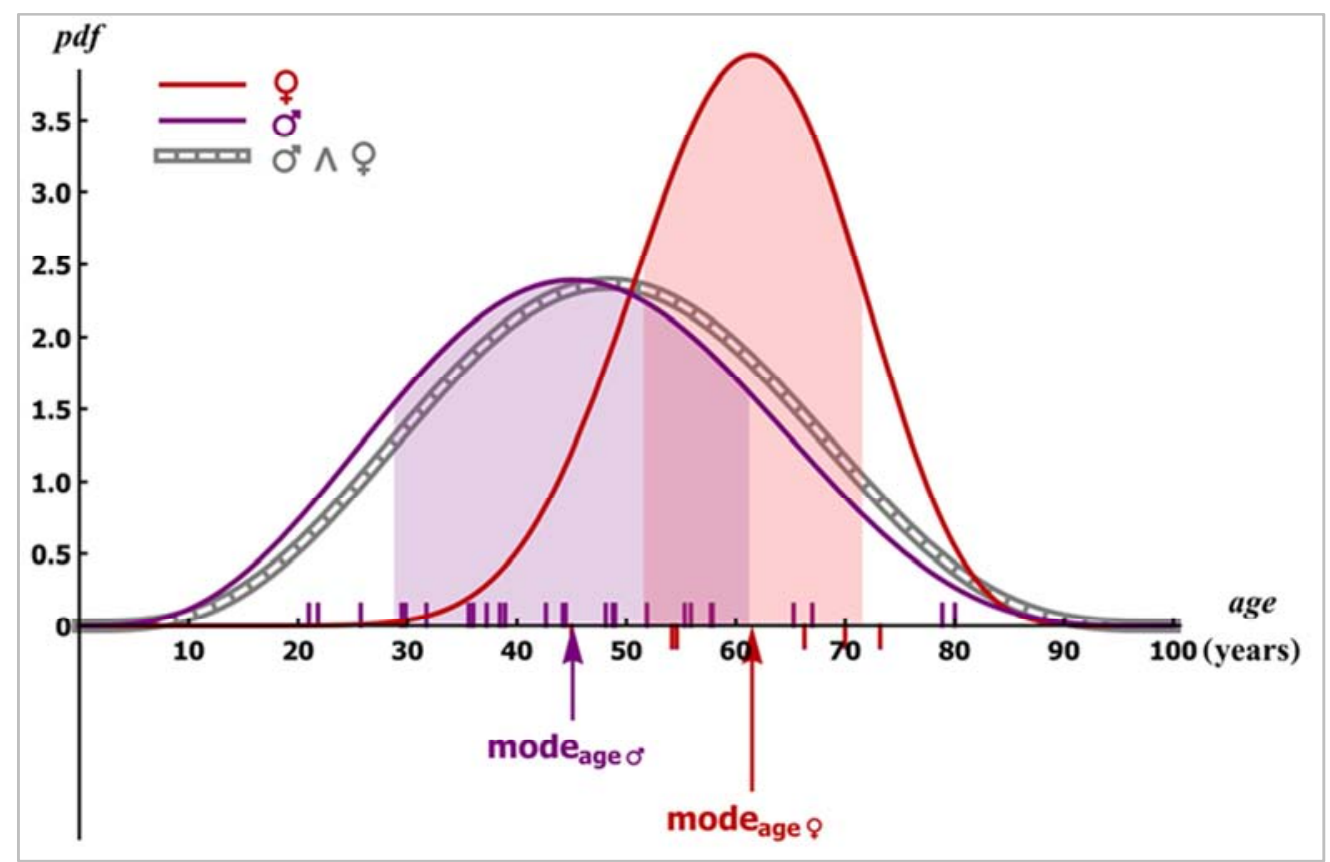

Figure 1. The pdfs (probability density functions) of the ML (most likely) populations (beta-distributed in all three cases) from which the samples had been drawn: purple (male), red (female), and gray (both sexes pooled). The colored, short vertical lines along the abscissa show the actual ages that have been rescaled to centuries from the patients'ages (in days, not in years) as described in the text. Arrows designate the modes and the colored areas are the $\pm 34.1 \%$ intervals about the modes. The ladder-type band is the ML Beta distribution when the sexes are pooled. Wilks' $\Lambda$ shows that the segmentation of the data set into two sets (populations) is not significant (at 5\% significance level), hence there is no need to calculate a confusion matrix or the area under the ROC curve. The ML Beta distribution for the males and females pooled shows a mode close to the mode for the males.

\subsubsection{D-Clustering of Each Parameter}

As a first step, we use one-dimensional $K$-means clustering [20] for ICP and TEMP components of the triple separately. We determine the boundaries between the clusters of each physiological parameter along its respective axes, fractions of occurrences per cluster, and the centroids (arithmetic means) for each of the clusters.

\subsubsection{Clustering of Parameter Triples in 3D}

We then use the three-dimensional $K$-means clustering algorithm $[20,21]$ to determine three clusters of the ICPTEMP-PSI triples. As we communicate in greater detail below, the gap between Cluster 3 and Clusters $1 \& 2$ is close to planar and the gap between Cluster 1 and Cluster 2 is planar and perpendicular to the ICP-TEMP coordinate axes plane (and therefore parallel to the PSI axis).

\subsubsection{Numerical Analyses of Gaps Between Clusters}

To find a planar approximation to the gap between Cluster 3 and Clusters $1 \& 2$, we find points on the convex hulls of the clusters that are closest to the convex hulls of the other clusters and then determine a least-squares (LSq) fitting plane to these points (via PCA, not via LSq regression). The intersection of this plane with the ICP-TEMP coordinate axes plane determines the functional dependence of $\mathrm{ICP}_{\text {threshold }}$ on temperature. Likewise, it informs the ICU therapist of possible functional dependence of PSI on $\mathrm{ICP}_{\text {threshold }}$.

\section{Results}

\subsection{Homogeneity of Ages of Patients}

Table 1 lists the modes, the expectations values, the $\pm 34.1 \%$ uncertainties and the ranges of the ages of the female and male patients that had suffered TBI and were treated in the ICU. The distribution of the population from which the female sample has been drawn is not significantly different from that of the male sample $(0.052>P>0.051$ using Wilks' $\Lambda)$. The log-likelihood ratio used in this way is in lieu of statistical power analysis of separation of the sexes.

\subsection{Boundaries of Clusters of Each Parameter}

Table 2 shows the boundaries and the fraction of occurrences in the case of clustering of the TEMP, the PSI and the ICP of the triples independently. We note that the ICP intervention boundary - $16.5 \mathrm{~mm} \mathrm{Hg}$ - is remarkably less than the $20 \mathrm{~mm} \mathrm{Hg}$ conventionally adopted in the published literature. [6] Whether the boundaries of TEMP (as they are in the vicinity of the mean body temperature of $37^{\circ} \mathrm{C}$ ) are diagnostically relevant is difficult to assess, [22] but these 
boundary values indicate that the clustering algorithm can be considered reliable.

Table 2. Listing of the diagnosed variables and their ranges for the patients included in this study.

\begin{tabular}{ll}
\hline Variable & Description of diagnosed variable \\
\hline ICP & Intracranial Pressure $[9]$ \\
& Observed Range: 0 to $+42(\mathrm{~mm} \mathrm{Hg})$ \\
TEMP & Body temperature $[12]$ \\
& Observed Range: $34.6^{\circ} \mathrm{C}$ to $38.5^{\circ} \mathrm{C}$ \\
PSI & Patient state index $[15]$ \\
& Observed Range: 6 to 100 (scale: $0-100)$ \\
\hline
\end{tabular}

Table 3. Boundaries between and frequencies of occurrences within clusters of ICP and TEMP singly. Only components of complete ICP, TEMP and PSI triples were used, albeit, for this table, the one-dimensional clustering algorithm was applied to each variable separately.

\begin{tabular}{llllll}
\hline Variable & Cluster 1 & ${\text { Boundary } \text { Cluster }_{1} \leftrightarrow \text { Cluster }_{2}}$ & Cluster 2 & ${\text { Boundary } \text { Cluster }_{1} \leftrightarrow \text { Cluster }_{2}}$ & Cluster 3 $^{3}$ \\
\hline ICP $(\mathrm{mm} \mathrm{Hg})$ & $n_{1}=498(50.6 \%)$ & 6.75 & $n_{2}=376(38.2 \%)$ & 16.5 & $n_{3}=110(11.2 \%)$ \\
TEMP $\left({ }^{\circ} \mathrm{C}\right)$ & $n_{1}=231(23.5 \%)$ & 36.15 & $n_{2}=476(48.4 \%)$ & 37.15 & $n_{3}=277(28.2 \%)$ \\
\hline
\end{tabular}

\subsection{Boundaries and Gaps of Clusters of Parameter Triples}

Table 4 shows the fractions of triples in each cluster, as well as the extremes of the convex hulls of the 3D-clusters (Figure 3a). The projected extremes can (and do) overlap because the gaps between the convex hulls need not be planes, let alone planes parallel to the planes formed by the coordinate axes. The gaps between the convex hulls are thresholds. The gap between Cluster 1 and Cluster 2 is indeed a plane which is perpendicular to the TEMP axis, at a temperature of $36.5^{\circ} \mathrm{C}$, thus indicating that there exist two clusters of ICP-TEMP-PSI triples below the ICP intervention threshold, and they are separated at $36.5^{\circ} \mathrm{C}$ (one publication [22] discusses hypothermia), as expected by clinicians. There is no gap along the TEMP axis above the ICP threshold gap. The result of the clustering algorithm shows that, above ICP threshold, temperature is not an intervention indicator, let alone a necessary one.

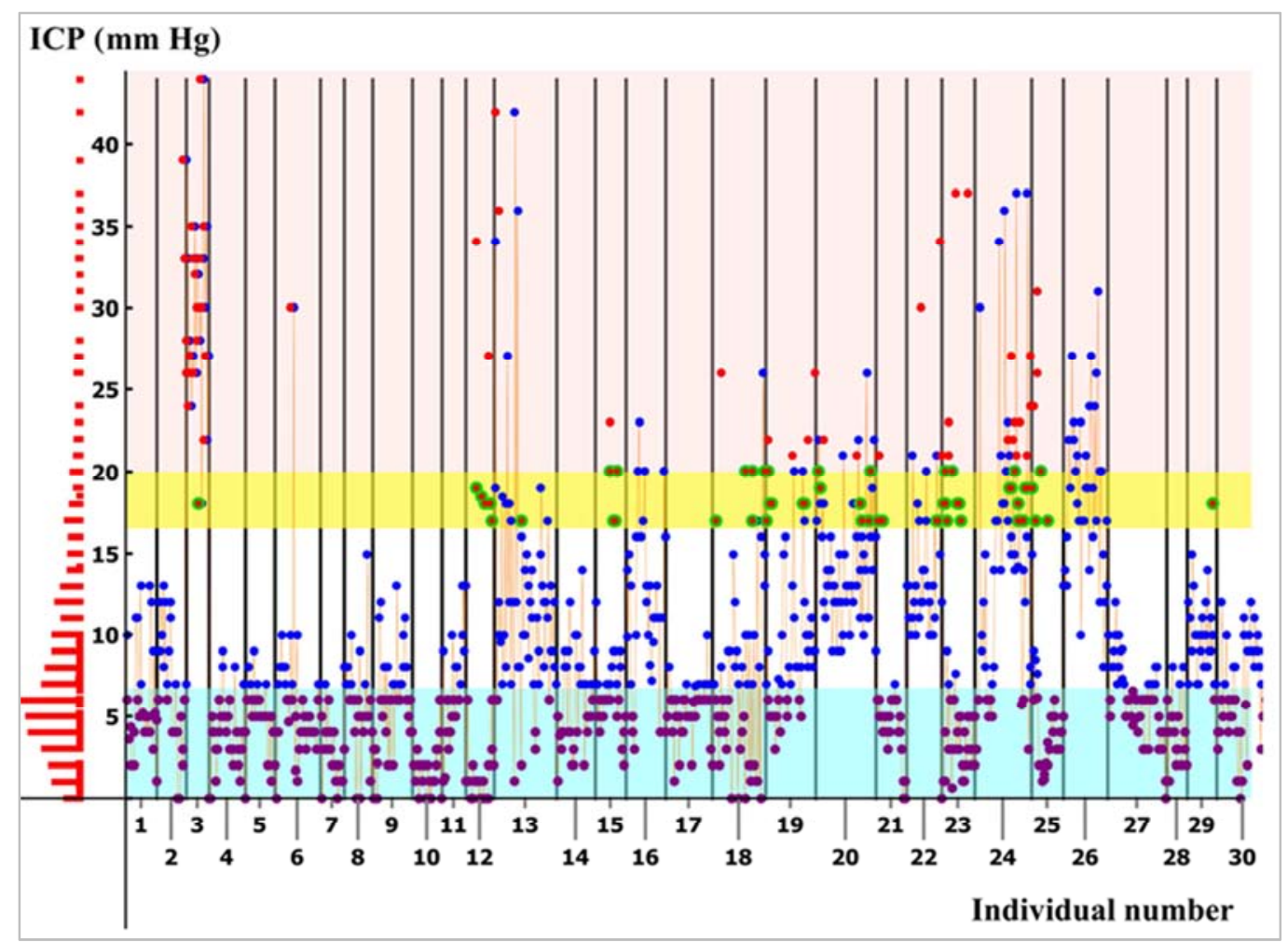

Figure 2. The complete ICP data set used for this study. Along the abscissa is the patient number. Data from each patient is within a strip bordered by thin vertical black lines. The points in every graph show registrations, but only those for which complete triples exist. The thin orange lines connecting the data points enhance the readability and suggest a time line; indeed, the leftmost point in each strip is the first registration and the rightmost point the last registration, but the spaces along the abscissa are not necessarily proportional to the time intervals between registrations (because of occasionally missing triples). To the left of the ordinate axis is the $\left(90^{\circ}\right.$ rotated) histogram of the registrations. Frequencies of occurrences, as well as boundaries, are listed in Table 3. The light yellow rectangle shows two boundaries: the upper boundary is the $20 \mathrm{~mm} \mathrm{Hg}$ intervention limit often referred to in the literature. The lower boundary is the cluster boundary found by one-dimensional K-means clustering, as described in the text. The green/red points are those data registrations where - we argue in this study - intervention should take place and would not warrant intervention if the traditional 20 mm Hg boundary were used in ICUs. 


\section{Discussion}

ICU clinicians dealing with TBI patients point out that the changes in the three parameters ICP, TEMP and PSI are driven by the trauma, and can be, to some extent, controlled by intervention. A change in the position of the ICP-TEMPPSI triple (Figure 4), therefore, alerts clinicians to whether intervention is called for. We, heeding this recommendation, based our statistical analysis on an algorithm for clustering these triples and we discovered, remarkably, that the triples do indeed cluster with medically interpretable gaps between the convex hulls; they, we argue, indicate thresholds. In other words, we did not devise a model with a particular threshold as a target parameter (neither for ICP, nor for TEMP, nor for PSI), but let the clustering algorithm find these gaps. The three clusters we found supply, among other insights, an intervention threshold for ICP.

The clustering algorithms applied to ICP and TEMP individually outputted two boundaries each, which we do not consider intervention thresholds (Table 3). The upper threshold for ICP is, however, notably lower than the recommended intervention threshold $(20 \mathrm{~mm} \mathrm{Hg})$ listed in the literature. We do not recommend this $16.5 \mathrm{~mm} \mathrm{Hg}$ threshold, however, because it is higher than the threshold found by the $3 \mathrm{D}$ clustering algorithm and because applying a clustering algorithm to the individual parameters separately implies the independence of intracranial pressure, temperature and sedation levels. Consequently, the outcome of the 3D clustering algorithm supports our rejection of $\mathrm{ICP}_{\text {threshold }}=16.5 \mathrm{~mm} \mathrm{Hg}$. Currently, we cannot present medical arguments for the boundary values along each parameter axis independently, nor are we aware of a discussion of such threshold values published elsewhere. What we do observe: if the 3 parameters are not considered independently, then we find meaningful, interpretable thresholds, which differ from the thresholds obtained using 1D clustering algorithms. In the context of the threshold outputted by the $3 \mathrm{D}$ clustering algorithm, we note that the one study that relates survival outcomes to ICP thresholds finds an asymptotic limit of $12-13 \mathrm{~mm} \mathrm{Hg}$ for the threshold for durations of high ICP exceeding $6 \mathrm{~h}$. [7] Although we do not look at the relationship between triples in Cluster 3 and survival outcomes (for two reasons: (a) because the number of non-survivors for this data set in our ICU is extremely small and (b) the clinicians at this ICU used $20 \mathrm{~mm} \mathrm{Hg}$ as a threshold - the analysis we present here is retrospective) we do point out that we did sample the ICP triples every 6 hours.

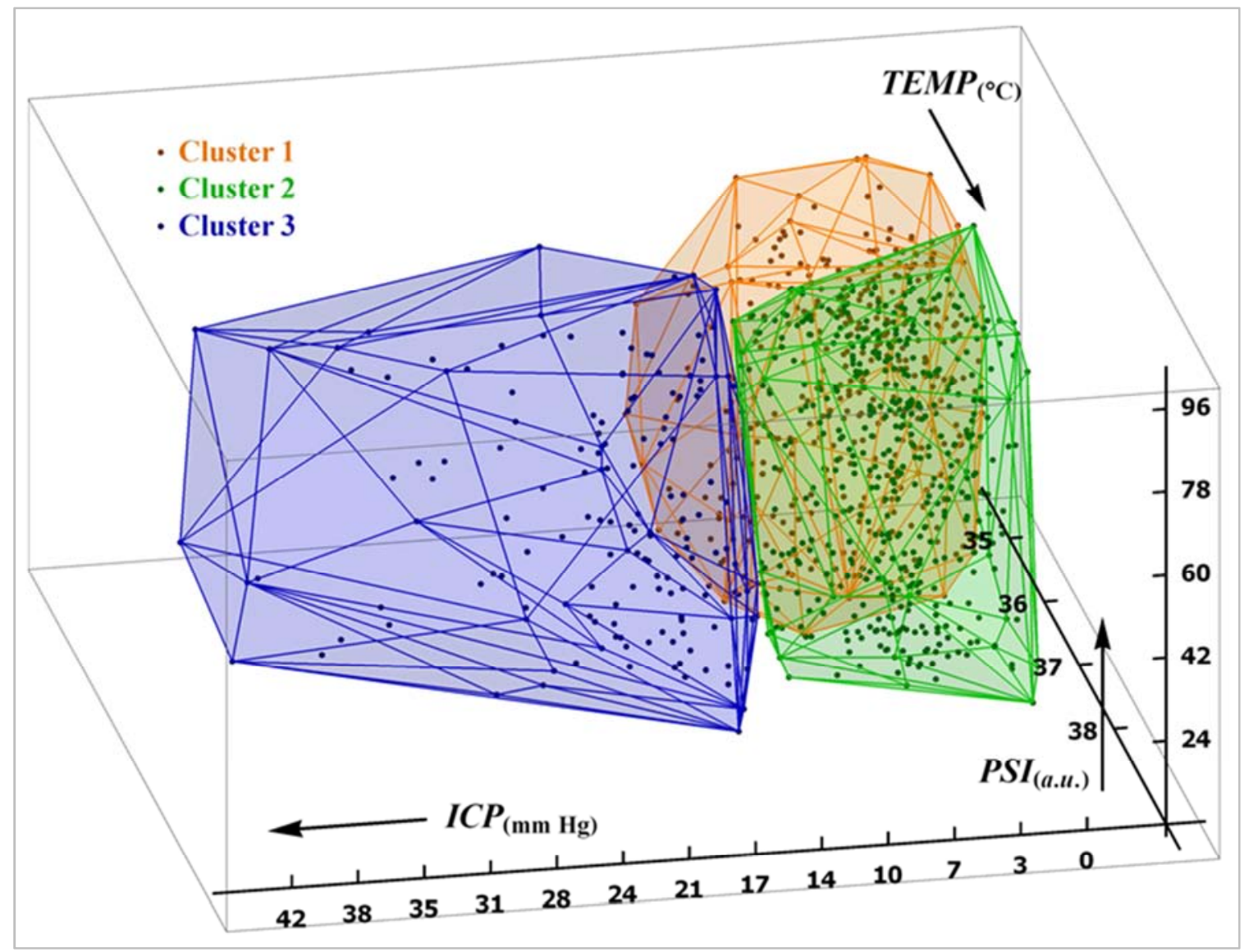

Figure 4. The graphical rendition of the outcome of the clustering algorithm applied to the triples (ICP, TEMP, and PSI) in 3D-space. There are three clusters separated by gaps. The convex hulls of these three clusters are shown as colored polyhedrons, and the triples as black points on and within these. Cluster 1 and Cluster 2 have a gap at constant temperature $\left(26.5^{\circ} \mathrm{C}\right)$; the gap terminates at the threshold for ICP. Cluster 3 is separated by an almost planar gap from Clusters $1 \& 2$. 


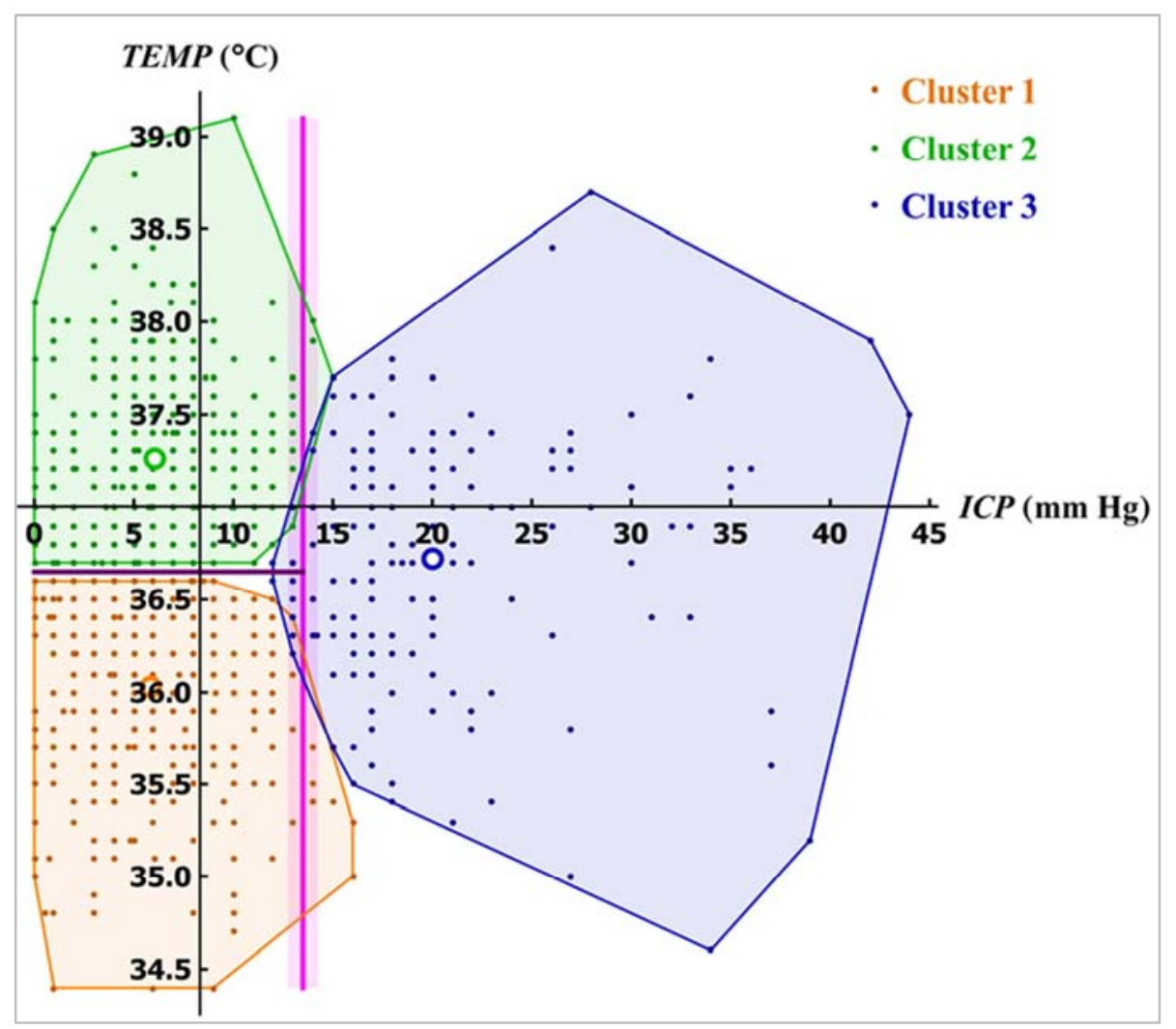

Figure 5. The polygons which are the projections on the ICP-TEMP plane of the clusters shown as polyhedrons in Figure 4. There is a clear separation between Cluster 1 and Cluster 2 perpendicular to the temperature axis. There is no critical temperature for ICP values in Cluster 3 - implying that temperature does not play a role in the necessity of intervention for ICP values above the intervention threshold of $13.625 \mathrm{~mm} \mathrm{Hg}$. In first (and very good) approximation, the intervention boundary for ICP is a projected plane. The uncertainty in the intercept of this plane on the ICP axis is shown as a band. The open circles mark the projections of the centroids of the clusters.

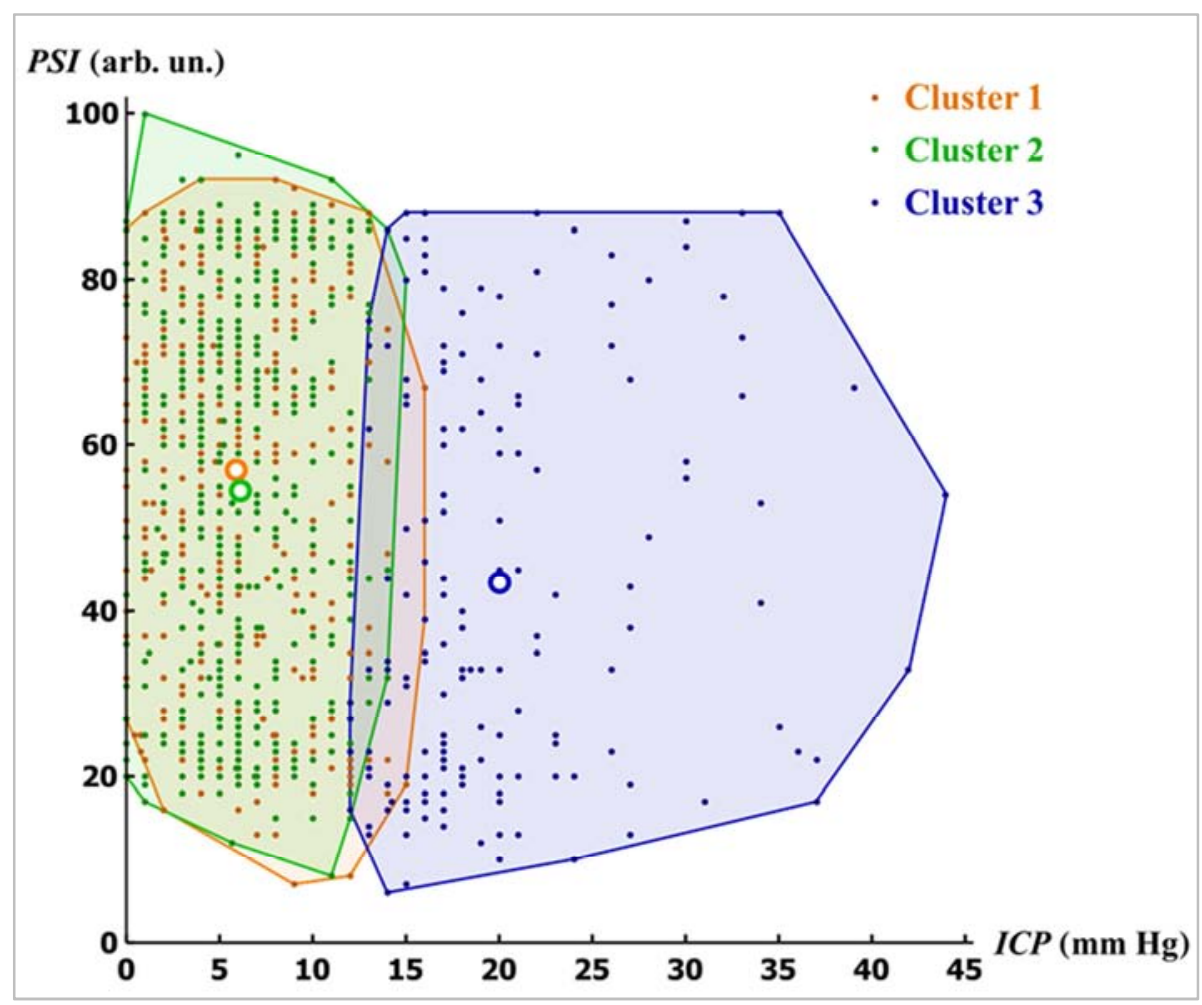

Figure 6. The projections of the polyhedrons shown in Figure 4 onto the ICP-PSI plane. There is a band/region separating Cluster 3 (the ICP registrations above the intervention threshold) from Clusters $1 \& 2$. In this projection, there is neither a gap nor a planar boundary separating clusters along the PSI axis. 


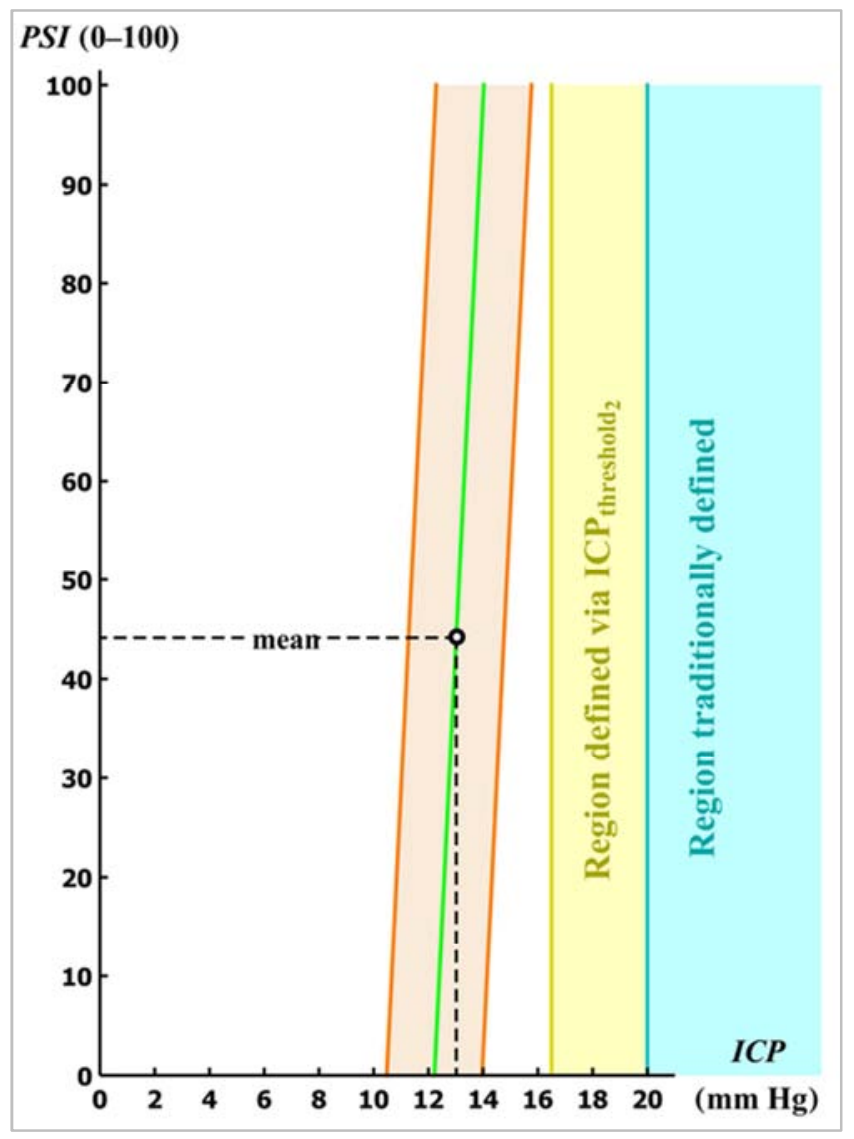

Figure 7. The illusionary PSI dependency on ICP intervention threshold, including uncertainty. One band (cyan) shows the realm of traditional/conventional threshold for ICP intervention $(20 \mathrm{~mm} \mathrm{Hg})$. The second band (light yellow) shows the shift in intervention threshold if onedimensional clustering of ICP is used (Figure 2). There is a further shift to a still lower ICP-intervention threshold if $3 D$ clustering is used. The leastsquares plane for the gap (compare Figure 4 and Figure 5) and its uncertainties appear as straight lines in the projection. We note the slope of this intervention line is extraordinarily steep (the axes' scales differ considerably), implying the ICP-intervention threshold is statistically independent of PSI. The change at the PSI endpoints in the projection is

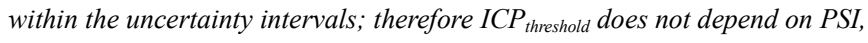
neither in a statistical sense, nor in medical relevance.

Segmenting a data set in three-dimensional space into three clusters does not ensure that boundary planes are generated, because the convex hull surfaces of each cluster (Figure 4) at the gaps are not necessarily planar. However, we discover that the one gap is indeed planar and the second one is very close to planar.

The temperature at the gap $\left(36.5^{\circ} \mathrm{C}\right)$ between Cluster 1 and Cluster 2 is considered physiologically normal by ICU clinicians (however, see a discussion [22]), while temperatures above or below this gap value call for interventions. We note that this gap was not inputted prior to the execution of the clustering algorithm; rather, the algorithm outputted this temperature gap value. This result of the clustering algorithm can be considered corroborating its reliability. We stress that the clustering algorithm does not output clusters that provide a temperature gap for ICP values above the ICP gap, which, we argue, is therefore an ICP intervention threshold value.

The second surprising result of this study is that the boundary between Cluster 3 and Clusters $1 \& 2$ is planar within statistical uncertainties and almost parallel to the TEMP and PSI axis, indicating that the ICP threshold for acute intervention is almost independent of PSI (Figure 7). The boundary between Cluster 1 and Cluster 2 is planar and perpendicular to the TEMP-axis, indicating that the temperature threshold is independent of PSI and independent of ICP for $\mathrm{ICP}_{\text {threshold }}<13.5 \mathrm{~mm} \mathrm{Hg}$.

The published literature refers (in the majority of studies) to an intervention threshold of $\mathrm{ICP}_{\text {threshold }}=20 \mathrm{~mm} \mathrm{Hg}$. $[4,6$, 23-24] The most recent review states "The most widely accepted ICP threshold for therapy is $20 \mathrm{~mm} \mathrm{Hg."} \mathrm{[6]} \mathrm{A}$ further review also mentions that ICP thresholds are based on "a body of experience" — thereby implying: not based on rigorous statistical analysis. [10]

Sampling and measuring ICP once every 6 hours suggests there may be a "hit or miss" source of error: ICP might increase rapidly shortly after the ICP has been registered and then decrease rapidly enough so that the ICP value may be innocuous by the time of the next registration. Per definition, we have no way of evidencing this effect directly, but we can offer a few observations that allow an estimation of whether this effect occurs. In one study, 155 measurements of ICP above $20 \mathrm{~mm} \mathrm{Hg}$ were registered in 200 patients with a measurement interval of 12 hours. [6] Here we registered 154 measurements in Cluster 3 for 32 patients with 6-hour registration intervals. Assuming the ICPs in Cluster 3 occur close to uniformly throughout 24 hours (Figure 2) for all patients combined, we should expect 77 measurements for 12-hour intervals ( 2 ICPs above threshold per patient). Our observed rate is comparable to [6] ( $80 \%$ ICPs above threshold per patient). However: the Cluster 3 threshold is, at $13 \mathrm{~mm} \mathrm{Hg}$, considerably lower and, furthermore, we do not observe ICPs above $20 \mathrm{~mm} \mathrm{Hg}$ in all patients (Figure 2). We do not, of course, know how often ICPs in [6] are above 13 $\mathrm{mm} \mathrm{Hg}$, nor whether any patients in [6] ever had ICPs above threshold, while some of our SHT patients did.

Although our analyses are rigorous, they do not address the issue of whether the ICP intervention threshold for young patients might differ from that of older patients - or that it might differ between the sexes. Despite the rather large number of data triples we use in this study, we caution against suggestions to split the data into young vs. old or male vs. female patients. (We have demonstrated - Figure 1 - that there is no observed age-related sexual dimorphism.) While every interpretation of data tries to identify statistical estimators that extract information from the noisy data set, there is a theoretical limit as to how much information can be extracted, given a prognostic model with likelihood $L$; this limit is Akaike's Information Criterion corrected for finite samples size $(A I C c)$, namely

$$
A I C c=-2\left(\ln \mathrm{L}-K \frac{n}{n-(K+1)}\right)
$$


where $K$ is the number of parameters in the model and $n$ is the sample size. $[25,26]$

The second term, called the "penalty term" [27], ensures that making a model more complex by increasing the number of parameters $K$ (and thereby also increasing the likelihood of the more complex model) does not include the modeling of statistical noise. If one were to partition the 984 triples into young versus old, say, then $\mathrm{L}$ might increase while $K$ stays constant for each age group or sex group. For example, by estimating the likelihood $\mathrm{L}$ with the same number $K$ of prognostic model parameters, but defining ML (maximum likelihood) distribution parameters for young and for old separately. But the penalty term increases nonlinearly with decreasing sample size $n$; so $A I C c$ need not decrease. If it does not, then modeling noise will have been included in the ML parameter estimation. [28] In particular, with the very noisy data set of ICP-TEMP-PSI triples we cannot assume that the likelihood L increases strongly enough (and it must increase exponentially, because its logarithm must outrun the nonlinear increase in the penalty term).

We agree with [6] that a single ICP threshold may not be optimal. But our analysis of insignificant age segmentation (Table 1 and Figure 1) shows that a considerably vast increase in data triples is necessary to statistically rigorously estimate target intervention thresholds that are age- or sexdependent - if at all. Our analysis therefore does not provide an indicator of the hypothesis in [6] being valid. Rather, we infer that the intervention threshold $\mathrm{ICP}_{\text {threshold }}$ seems to be age independent. In any case, the conventionally assumed intervention threshold of $20 \mathrm{~mm} \mathrm{Hg}$ is most probably far too high.

Much of the early literature (quoted in [3]) derives an $\mathrm{ICP}_{\text {threshold }}$ value based on indicators for reduction in mortality. [29] Here we look at the pattern inherent in the data - mortality rates may also change with our patternderived $\mathrm{ICP}_{\text {threshold }}=13 \mathrm{~mm} \mathrm{Hg}$ - but we would not infer that they would increase. Furthermore, reduction in mortality is not the only ICU goal; post TBI health is also an issue, for which no statistical analysis has yet been published.

The observed incline of the gap at ICP threshold relative to the PSI axis can be misleading (Figure 7). The change in $\mathrm{ICP}_{\text {threshold }}$ from PSI $=0$ to PSI $=100$ is less than the $\pm 34.1 \%$ interval overlaps because the slope is so steep. Consequently, there is no statistically rigorous dependence of $\mathrm{ICP}_{\text {threshold }}$ on PSI that is consequential for ICU clinicians.

\section{Conclusion}

The literature cited in the most recent reviews about ICP thresholds for intervention in the case of TBI list $20 \mathrm{~mm} \mathrm{Hg}$ as the recommended value. [6, 10, 30] The $20 \mathrm{~mm} \mathrm{Hg}$ threshold is based on 'conventional wisdom'. Here we presented a statistical analysis that supplies an intervention threshold, based on the results of a 3D clustering algorithm. We observed that a meaningful clustering algorithm may not be applied to a one-dimensional data set; i.e. the vital parameters (ICP, TEMP and PSI) cannot be regarded as physiologically independent. We found the intervention limit for ICP is much lower than the conventional $20 \mathrm{~mm} \mathrm{Hg}$ : namely, $16 \mathrm{~mm} \mathrm{Hg}$ (for clustering in 1D) and $13.625 \mathrm{~mm} \mathrm{Hg}$ (for clustering in 3D). Remarkably, the ICP threshold found by clustering in $3 \mathrm{D}$ is de facto independent of body temperature and patient state index, the proxy for degree of unconsciousness and/or sedation. The 3D clustering algorithm had no input parameters, only the 984 triples. It is noteworthy (and unexpected, from an algorithmic point of view) that the algorithm finds a planar gap perpendicular to the temperature axis, but only below the gap $\left(13.625\left\{\begin{array}{l}+1.5 \\ -1.4\end{array}\right.\right.$ $\mathrm{mm} \mathrm{Hg}$ ) which defines the lowest ICP values for Cluster 3. This gap is, within statistical fluctuations along the convex hull of Cluster 3, also planar and perpendicular to the other gap (Figure 6).

We argue, based on the analysis presented here, that the threshold for ICP, above which intervention is expedient, should be 13-14 $\mathrm{mm} \mathrm{Hg}$.

\section{References}

[1] M. Smith, (2008) Monitoring intracranial pressure in traumatic brain injury. Anesthesia and Analgetics 106, 240248 .

[2] M. Czosnyka, and J. D. Pickard (2004) Monitoring and interpretation of intracranial pressure. Journal of Neurological Neurosurgery and Psychiatry 5, 813-821.

[3] N. Stocchetti, A. Colombo, F. Ortolano. et al. (2007) Time course of intracranial hypertension after traumatic brain injury. Journal of Neurotrauma 24, 1339-1346.

[4] S. L. Bratton, R. M. Chestnut, J. Ghajar, et al. (2007) VIII. Intracranial Pressure Thresholds. Journal of Neurotrauma 24, Suppl. 1, 55-58.

[5] E. Sorrentino, J. Dredler, M. Kaspowicz et al. (2012) Critical thresholds for cerebrovascular reactivity after traumatic brain injury. Neurocritical Care 16, 258-266.

[6] N. Stocchetti, M. Carbonara, G. Citero, A. Ercole, M. B. Skrifars, P. Smielewski, T. Zaerle, and D. Menon (2017) Severe traumatic brain injury: targeted management in the intensive care unit. Lancet Neurology 16, 452-464.

[7] F. G. Guiza, B. D. Depreitere, I. P. Piper, G. V. Van den Berghe, and G. M. Meyfroidt (2014) New look at $20 \mathrm{~mm} \mathrm{Hg}$ ICP Threshold. Journal Critical Care 18 (Suppl 1), 458.

[8] N. Carney, A. M. Totten, C. O'Reilly et al. (2017) Guidelines for the management of severe traumatic brain injury. Fourth edition. Neurosurgery $80,6-15$.

[9] R. A. Weerakkody, M. Czosnyka, R. A. Trivedi, and P. Hutchinson P (2009) "Intracranial Pressure monitoring in head injury." in "Head Injury: A Multidisciplinary Approach", P. C. Whitfield, E. O. Thomas, F. Summers, M. Whyte, and P. J. Hutchinson, Eds. Cambridge, UK, Cambridge University Press.

[10] C. Lazaridis C et al. (2014) Patient-specific thresholds of intracranial pressure in severe traumatic brain injury. Journal of Neurosurgery 120, 893-900. 
[11] T. G. Saul and T. B. Drucker (1982) Effects of intracranial pressure monitoring and aggressive treatment on mortality in severe head injury. Journal of Neurosurgery 56, 498-503.

[12] W. C. Fallis (2002) Monitoring urinary bladder temperature in the intensive care unit: state of the science. American Journal of Critical Care 11, 38-45.

[13] P. L. Purdon, A. Sampson, K. J. Pavone et al. (2015) Clinical Electronencephalography for Anesthesiologists - Part I: Background and basic signatures. Anesthesiology 123, 937960.

[14] M. Oddo and L. A. Steiner (2016) Sedation and analgesia in the neurocritical care unit. Chapter 6 in, "Textbook of Neurocritical Care", M. Smith, G. Citerio, and W. A. Kafka, Eds. Oxford, UK, Oxford University Press, pp. 65-77.

[15] D. R. Drover, H. J. Lemmens, E. T. Pierce, G. Plourde, G. Lloyd, E. Ornstein, L S. Prichep, R. J. Chabot, L. Gugino (2002) Patient state index: Titration of delivery and recovery from Propofol, Alfentanil, and Nitrous Oxide anesthesia. Anesthesiology 97, 82-89.

[16] S. Vacas, E. McInrue, M. A. Gropper, M. Maze, R. Zak, E. Lim, J. M. Leung (2016) The feasibility and utility of continuous sleep monitoring in critically ill patients using a portable electroencephalography monitor. Anesthesiology and Analgetics 123, 206-212.

[17] S. S. Wilks (1937) The large-sample distribution of the likelihood ratio for testing composite hypotheses. Philosophical Transactions of the Royal Society A 231, 60 62.

[18] J. P. Huelsenbreck and K. A. Crandall (1997) Phylogeny estimation and hypothesis testing using maximum likelihood. Annual Review of Ecological Systems 28, 437-466.

[19] H. Prossinger and F. L. Bookstein (2003) Statistical estimators of frontal sinus cross section ontogeny from very noisy data. Journal of Morphology 257, 1-8.

[20] D. J. C. MacKay Information Theory, Inference, and Learning Algorithms, 2004, Cambridge, UK, Cambridge University Press.
[21] M. Bishop (2006) "Pattern recognition and Machine Learning", New York, NY, USA, 2006, Springer.

[22] P. J. Andrews, H. L. Sinclair, A. Rodriguez et al. (2015) Hypothermia for intracranial hypertension after traumatic brain injury. New England Journal of Medicine 373, 2403-24012.

[23] N. Stochetti and A. I. Maas (2014) Traumatic intracranial hypertension. New England Journal of Medicine 370, 2121 2130.

[24] Le Roux P (2016) "Intracranial pressure monitoring and measurement." Chapter 15 In: "Translational Research in Traumatic Brain Injury." D. Laskowitz, and G. Grant, Eds. Boca Raton (FL): CRC Press/Taylor and Francis Group, 2016.

[25] H. Akaike (1973) "Information theory as an extension of the maximum likelihood principle." In: "Second International Symposium on Information Theory." B. N. Petrov, and F. Csaki, Eds. Budapest, Hungary, Akademiai Kiado.

[26] Takeuchi K (1976) Distribution of informational statistics and a criterion of model fitting. Suri-Kagaku (Mathematical Sciences) 153, 12-18. (In Japanese)

[27] K. P. Burnham and D. R. Anderson (2002) "Model Selection and Multimodel Inference: A Practical Information-Theoretic Approach.” $2^{\text {nd }}$ edition. New York, NY, USA, Springer.

[28] H. Akaike (1981) Likelihood of a model and information criteria. Journal of Econometrics 16, 3-14.

[29] F. Guiza, I. Piper, G. Van den Berghe, G. Meyfroidt (2013) Novel methods to predict increased intracranial pressure during intensive care and long-term neurological outcome after traumatic brain injury: Development and validation in a multicenter dataset. Neurological and Clinical Care 41, 554564.

[30] A. I. R. Maas, D. K. Memon, P. D. Adelson, et al. (2017) Traumatic brain injury: integrated approaches to improve prevention, clinical care, and research. Lancet Neurology 16, 987-1048. 